\title{
Interactions Between Spacecraft and Their Environments
}

Dr. Dale C. Ferguson

Lewis Research Center

Cleveland, Ohio

Prepared for the

31st Aerospace Sciences Meeting and Exhibit

sponsored by the American Institute of Aeronautics and Astronautics

Reno, Nevada, January 11-14, 1993

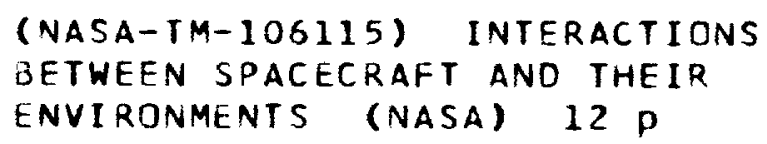


$+$

. 


\title{
INTERACTIONS BETWEEN SPACECRAFT AND THEIR ENVIRONMENTS
}

\author{
Dr. Dale C. Ferguson" \\ NASA Lewis Research Center \\ Cleveland, Ohio
}

\begin{abstract}
Spacecraft inevitably interact with their environments. Besides the interactions one immediately thinks of in space (zero-g, solar heating, atmospheric drag, expansion into vacuum conditions, etc.) other interactions are also important. Those of interest to spacecraft designers so far may be grouped under several headings; plasma interactions and spacecraft charging, impacts of debris and micrometeoroids, chemical reactions with neutral species, radiation degradation, etc. Researchers have made great progress in defining and evaluating the interactions of spacecraft with their expected ambient environments near Earth and in interplanetary space. Here we discuss some of these interactions with an eye toward expanding our knowledge into new environments, such as may be found at the moon and Mars, that will interact in new and different ways with exploring spacecraft and spacefarers.
\end{abstract}

\section{Introduction and Overview}

Interactions of spacecraft with their environments have traditionally been important in terms of reliability. The Van Allen radiation belts were discovered from saturation (temporary shutdown) of a Geiger counter ${ }^{1}$ on Explorer $I$. Anomalies in spacecraft operation in geosynchronous orbit have often been traced to spacecraft charging and arcing during solar substorm events. The Marecs-A spacecraft experienced a power loss on part of its solar arrays due to such a malfunction ${ }^{2}$. Spacecraft encountering the powerful radiation belts of Jupiter have undergone single event upsets (SEUs) due to their hard

'Physicist, Space Environment Effects Branch.

Copyright 1993 by the American Institute of Aeronautics and Astronautics, Inc. No copyright is asserted in the United States under Title 17, U.S. Code. The U.S. Government bas a royalty-free license to exercise all rights under the copyright claimed herein for Govemment purposes. All other rights are reserved by the copyright owner. radiation environment. Solar cells for space applications are routinely covered with coverslips to reduce radiation damage.

More recently, high voltage spacecraft in the low Earth orbit (LEO) environment have experienced significant interactions with their environment. The Upper Atmospheric Research Satellite (UARS), with a power system providing $100 \mathrm{~V}$ when coming out of eclipse was seen to charge to $90 \mathrm{~V}$ negative of its surrounding plasma because of plasma interactions with its solar arrays ${ }^{3}$. This has made data interpretation from some of its instruments difficult. Because Space Station Freedom would float (it has been estimated) $140 \mathrm{~V}$ negative of its surrounding plasma, and thus would be liable for sputtering and arcing damage, a plasma contactor to control its floating potential has been baselined ${ }^{4}$.

Other spacecraft also will interact with their environments, and this must be considered in designing payloads, missions, etc. The types of interactions they will undergo depend on the environments to be encountered in their missions. Many good papers have been written about spacecraft environmental interactions in different regimes, so this paper will deal only in generalities.

In the GEO (Geosynchronous Earth Orbit) environment, the major interaction of concern is differential charging of different parts of a spacecraft, leading to high electric fields and arcing between spacecraft components's. In GEO, the ambient plasma thermal current densities are insufficient to discharge spacecraft surfaces rapidly. Although there are many sources of charging (i.e., the photoelectric effect, secondary electron emission, etc.) most arcs are the result of charging during solar substorm events, when the Earth's geomagnetic tail, laden with particles from a sudden solar wind transient, undergoes field line breakage and reconnection, accelerating electrons to 
thousands of electron volts. These electrons charge insulating spacecraft surfaces, increasing electric fields to adjacent conductors beyond a breakdown level, leading to arcing ${ }^{6}$. The resultant arc currents, travelling through spacecraft conductors, can upset electronic components, induce spurious signals, etc. A common design solution for GEO is to coat all outside spacecraft surfaces with conducting materials, to eliminate the possibility of differential charging?

In LEO, absolute charging of spacecraft surfaces with respect to the surrounding plasma is of great concern. Because LEO thermal plasma current densities are high, surfaces do not ordinarily differentially charge, and total absolute potentials are bled off rapidly by collected plasma currents. However, some spacecraft impose differential charging on themselves by using distributed high voltages. Efficient power distribution requires either high voltages or massive conducting cables ${ }^{8}$, and most designers have chosen the former option. Spacecraft surfaces float at potentials that result in no net current collection from the plasma. If conductors at different voltages are not exposed to the space plasma, all spacecraft surfaces will float within a few volts of the surrounding plasma potential. If conductors of similar area but at different voltages are exposed to the plasma, a rule of thumb is that the most negative surfaces will float negative of the plasma about $90 \%$ of the total voltage difference between the surfaces. This is true, for instance of solar arrays, where interconnects between solar cells or cell edges are exposed to the plasma, yet operation of the array depends on the voltage distribution. Even some spacecraft designs without solar arrays place different surfaces at different potentials. For instance, a payload of the proposed SP-100 space nuclear power system will likely float about $100 \mathrm{~V}$ negative of a LEO plasma (see Figure 1, from Ref. 9) because the structure ground is discontinuous between power supply and payload. Locally, insulating surfaces will charge only a few volts negative of the surrounding plasma, however.

Arcs in LEO can occur from conductorinsulator junctions (including holes in cable insulation) when the conductor is highly negative of the surrounding plasma, or from anodized or other dielectric surfaces when the underlying conductor is at a negative potential higher than the dielectric breakdown strength of the coating ${ }^{10}$. Arc currents may flow out into the surrounding plasma, with the return currents distributed over wide areas of other spacecraft surfaces. Arcs also can occur through the plasma between closely spaced conductors at differing voltages ${ }^{11}$. Conducting surfaces highly negative of the plasma will attract high energy ions, and will be liable to sputtering ${ }^{10}$. Nearby surfaces may acquire a sputtered conducting coating, changing their electrical, optical, and thermal properties. At high positive potentials (if they occur), electrons will be collected, leading to localized heating and significant power drains ${ }^{12}$. Conductors may become exposed by micrometeoroid and/or debris impact, dielectric breakdown, etc.

The neutral spacecraft environment is also important. Chemically active species, such as the predominating atomic oxygen of LEO, can oxidize and damage surfaces, especially in the ram direction, where the spacecraft ram velocity simulates a high energy beam. Volatile oxidation products may be lost, leaving a surface denuded of its protective coverings. Chemically active ions may be attracted by charged spacecraft surfaces, and their reaction rates increase with energy ${ }^{13}$. Such considerations are important mainly for low planetary orbits such as LEO, but also may be important in low Mars orbit.

In LEO, radiation from the Van Allen belts may damage electronics and lower the output of solar cells with prolonged exposure. In addition, micrometeoroid and debris impacts may puncture insulators, pressure vessels and manned compartments unless they are accounted for in spacecraft design.

In low pressure neutral atmospheres, such as on the Martian surface and areas surrounding lunar bases, other interactions such as Paschen breakdown of atmospheric gases and arcing from dusty surfaces may be important ${ }^{14}$.

In this paper, Space Station Freedom (SSF) and the SP-100 power system will be used as examples of spacecraft now nearing the end of their design phase and how they may interact with their environments when completed. 


\section{Grounding Schemes and Surface Potentials}

The electrical grounding scheme of spacecraft is important in determining the spacecraft structure's potential, relative to the surrounding plasma. Because Space Station Freedom has the structure ground point at the potential of the most negative end of its solar arrays, which are producing a $160 \mathrm{~V}$ distributed voltage, SSF would float about $140 \mathrm{~V}$ negative of its surroundings ${ }^{10}$. To control its floating potential, a plasma contactor has been baselined, to keep all points of the structure within $40 \mathrm{~V}$ of the surroundings. For a large spacecraft such as SSF, even its velocity through the Earth's magnetic field induces significant potentials on its structure. It may be calculated, for instance, that there may be a $20 \mathrm{~V}$ potential difference, relative to the surrounding plasma, from one end of SSF to the other. SSF lies at one extreme; a large solar array powered spacecraft.

At the other extreme lies the SP-100 power system. Because it is nuclear powered, no high distributed potentials must contact the plasma in the SP-100 design. Also, it is small, compared to SSF. Therefore, one would expect SP-100 to "float" near to the surrounding plasma potential. However, SP100 power module has a $200 \mathrm{~V}$ total system voltage, divided into two halves, with the structure ground in the middle $e^{15}$. At the User Interface Module (UIM), the payload is connected to the power system. The payload sees a full $200 \mathrm{~V}$ difference, and is grounded to the negative end of the power supply. Drawings show a connection between the SP-100 structure ground and the payload structure ground through a resistance (see Figure 2, adapted from Ref. 15). As presently configured, to prevent a very large power loss in the resistor, the resistor's value must be high enough that the payload structure ground be $100 \mathrm{~V}$ negative of the SP-100 structure ground. In the UIM, where the structures are attached, there thus will be a $100 \mathrm{~V}$ difference in the structure potentials. Attached structures must be connected with an insulating material of sufficient thickness to stand off the $100 \mathrm{~V}$ difference. However, the surrounding plasma also will have a small region of high electric fields (a sheath). The NASCAP/LEO and POLAR computer codes have shown that local electric fields at and near the User Interface Module will be high'. Thus, for the SP100 design, particular attention must be paid to geometries and materials in the UIM region, to prevent arcing at conductor-insulator junctions in LEO. In particular, conductors known to have a plasma arcing threshold higher than $100 \mathrm{~V}$ must be used.

Materials commonly used in solar cells, such as silver coated interconnects and silicon, do not arc at voltages less than ${ }^{6}$ about $200 \mathrm{~V}$. Copper, on the other hand, has been inferred (from ground plasma tests) to arc at a much lower voltage (possibly" as low as $40 \mathrm{~V}$ ). Arcing thresholds for other materials have not yet been determined. There is some evidence that arc rates are higher for materials that absorb and/or adsorb water or other volatile materials ${ }^{16}$. Such materials (including some types of adhesives and polymeric materials) should be avoided at conductor-insulator junctions. Geometry can influence plasma arcing as well, and geometries that help prevent ions from entering the conductor-insulator region are preferred. The SPEAR-I rocket flight avoided arcing with voltages much higher than $100 \mathrm{~V}$ by clever exclusion of plasma ions from conductor-insulator junctions ${ }^{17}$. Designs to do this require extensive computer calculations of particle orbits in the anticipated geometries and electric fields using codes such as NASCAP/LEO.

\section{Floating Potentials}

While in general, spacecraft will take on floating potentials to maintain collected zero net current to exposed surfaces, NASCAP/LEO and EPSAT computer models have shown that payloads or structures on some spacecraft will float highly negative of the LEO plasma. In the case of the SP-100 power system, the payload floats highly negative because of the large amount of exposed conductor in the power system, which will effectively ground the power system to the surrounding plas$\mathrm{ma}$, and the peculiar grounding scheme used on SP100 , which places the payload $100 \mathrm{~V}$ negative of the power system. The small payload area then must collect a current of sluggish ions to balance the current of mobile electrons easily collected by the large power system area. The power system will float near the plasma potential, pushing the payload far negative. 
On solar array powered spacecraft such as SSF, a low voltage system will float near the plasma potential. A high voltage positively grounded power system will have parts of the solar arrays at high negative potentials, but the structure will float near the plasma potential. Again, this is because of the large conducting surface area available to collect the more mobile electrons. A negatively grounded power system, on the other hand, will make the structure work to collect the sluggish ions, and even the large surface area may only shift the floating potential away from the most negative system voltage by a few volts. On the face of it, then, high voltage solar array powered spacecraft for use in LEO may control their floating potentials by using a positively grounded power system. However, space qualified high voltage electronics of this polarity are scarce, slow, and may be prohibitively expensive.

For LEO missions, structure and payload surfaces at high negative potentials should avoid exposed conductors, or at least exposed conductorinsulator junctions, to avoid the same sort of arcing considered above for the SP-100 UIM joint. For GEO missions, fully conductive surfaces should be used everywhere to avoid differential charging?

\section{Dielectric Breakdown}

Ground tests and modeling done for the Space Station Freedom Electrical Grounding Tiger Team effort found that dielectric coatings often break down at $-100 \mathrm{~V}$ in a LEO plasma ${ }^{18}$. Rated dielectric strengths and strengths measured in an atmosphere were not consistently reached before breakdown in the plasma. It is suspected that this is due to a porosity of the coatings, allowing plasma ions to reach much closer to the underlying conductor than the nominal dielectric coating thicknesses. Even coatings with rated dielectric strengths of much more than $100 \mathrm{~V}$ seemed porous enough to the plasma ions to have real dielectric strengths of less than $100 \mathrm{~V}$. Thus, for surfaces that must be exposed at high negative potentials, it is important that dielectric coatings used on its outer surfaces be strong enough to stand off the full system voltage in a plasma. Care must be used in selecting surface coatings of high dielectric strength. In particular, the chromic acid anodization commonly used on aluminum exposed to space is often of insufficient strength to stand off $100 \mathrm{~V}$ in a plasma. Still, thickening the coating usually influences its thermal properties, so a thicker coating may not be a good answer. Sulfuric acid anodized coatings have greater dielectric strengths, but very different thermal properties. For LEO missions, it is recommended that sulfuric acid anodization be used in regions where thermal control is not an overriding concern, and that $A O$ protected aluminized kapton blankets (kapton surface on the outside) of $1000 \mathrm{~V}$ dielectric strength be used in all other exposed high voltage surfaces.

\section{Micrometeoroids and Debris}

The micrometeoroid and debris environment must be considered when designing pressurized vessels, fluid systems, etc. Studies of the expected flux of debris and micrometeoroids of a certain size are uncertain, due to the uncertainties in the amount of spacecraft debris that will be produced, models of its collision and breakup into smaller fragments, and models of the atmospheric drag that will eventually remove it from orbit ${ }^{19}$. While SP-100 had enough redundancy built in to survive collisions with $\mathrm{cm}$ size debris particles, SSF requires a meteoroid and debris shield to guarantee the lifetimes of its pressurized manned modules against impacting debris particles up to $1 \mathrm{~cm}$ in size.

\section{Radiation}

The radiation environment includes both ionizing electromagnetic radiation from the sun (UV, X-rays, etc.) and energetic charged particles from solar flares and the solar wind, the Van Allen belts $^{20.21}$, and cosmic rays. Solar UV may cause color changes in paints and breakdown of polymers. Charged particle bombardment in the Van Allen belts has led most spacecraft designers to shun orbits within the belts, but even in LEO and GEO, spacecraft must be designed to withstand the radiation environment. Of course, one of the main concerns of manned missions to Mars and elsewhere in the solar system is the possibility of irradiation by a strong solar flare. In LEO, SSF will be protected from the direct effects of solar flares and most cosmic rays by the Earth's magnetic field. 
Nevertheless, it must use coverslides to protect its solar cells from degradation, and non-yellowing paints and surface coatings. SP-100 creates its own radiation hazard, and its payloads must be shielded from its nuclear reactor and placed at a distance from the power source. On the Moon and Mars, the radiation environment is usually similar to that in interplanetary space, with no strong magnetic fields for shielding, and no dense atmosphere to block solar UV and X-rays.

\section{Sputtering}

Sputtering may also be a concern for long duration missions in LEO with surfaces at high negative voltages. A complicating factor is that in LEO, the sputtering species is atomic oxygen, for which little information is known, and for which chemical effects may contribute to sputtering rates. Much work has been done on a sputtering model to help evaluate surface material loss rates in $\mathrm{LEO}^{2}$. Measurements of low energy sputtering by atomic oxygen have been made by Eck and others at CWRU. Out of these measurements has come a theory of low energy sputtering which better agrees with the measurements than previous theories ${ }^{23}$. One distinguishing characteristic of the theory is that there is no absolute threshold for sputtering, but the rate at low energies is determined by the thermal tail of the surface material atom velocity distribution.

Sputtering rates are a strong function of the surface voltage. Because the voltages on spacecraft are usually not in the kV range (which would imply high sputtering rates) sputtering will only be important for mission times as long as years in a high density plasma, such as that in LEO or low Mars orbit. Sputtering may also be a long-term problem in the lunar environment, if locally produced environmental plasmas have a high enough density. Whenever long life is required in a high density plasma environment, surfaces should be insulated to prevent direct contact of high potential conductors with the plasma.

The SSF Electrical Grounding Tiger Team showed that where micrometeoroids, debris, or manufacturing defects produce small pinholes in insulators, the sputtering rate will be greatly in- creased by ion focusing onto the pinhole ${ }^{24}$. Underlying conductors must be of sufficient thickness to withstand puncture at accelerated sputtering rates (see Figure 3, adapted from Ref. 25). Materials of low sputtering rate also may be used, if they satisfy thermal, atomic oxygen, and other requirements. Optical, thermal, or other specialized coatings in the line of sight to the sputtering pinhole will become coated with the sputtered material over a long period of time. It is desirable to place such surfaces so they have no direct line of sight to surfaces that might undergo sputtering.

\section{Parasitic Power Drain}

Wherever biased exposed conductors exist, plasma currents will be collected. Parasitic power losses due to plasma current collected from pinholes or coating defects have been quantified and shown to be small in ground plasma chamber testing at LeRC. In particular, Grier and Domitz $z^{26}$ tested several candidate cable insulating materials; and showed that below a few hundred volts positive, collected currents remained negligible. At voltages of $200 \mathrm{~V}$ and above, insulation pinholes showed snapover effects. If conductors are at high positive potentials relative to the plasma, snapover may greatly increase the electron currents collected, and the resulting power drain. Still, regardless of spacecraft grounding scheme, it is unlikely that spacecraft conductors will be at potentials greater than $100 \mathrm{~V}$ above the plasma potential, so snapover is unlikely to occur. An exception is SSF. Here, the plasma contactor that will keep the structure close to plasma potential will push the most positive end of the solar arrays more than $100 \mathrm{~V}$ positive, and the solar cell edges will collect more current than otherwise, essentially increasing the power drain and demanding a high current capacity for the plasma contactor.

For other spacecraft, such as SP-100, NASCAP/LEO modeling has shown the power loss from currents to other surfaces is small compared to the total delivered current, and thus the percentage efficiency loss is also small?. A rule of thumb is that for every square meter of exposed conductor in LEO a parasitic structure current of about $1 \mathrm{~mA}$ may be expected. Thus, for a payload of about 100 square meters surface area (on SP-100, for instance) 
only $100 \mathrm{~mA}$ of structure current may be extracted from the power supply capacity, compared to the 500 A that the SP-100 power source may deliver at $200 \mathrm{~V}$ and $100 \mathrm{~kW}$.

\section{Atomic Oxygen Durability}

Materials exposed to atomic oxygen (AO), the predominant species in LEO, will be subject to rapid oxidation. In contrast to sputtering by ions in LEO, where the sputtering rate per incident particle may be 0.1 and the flux of sputtering particles on the order of $10^{12}$ per square centimeter per second, atomic oxygen reaction rates may be 1.0 and the ram flux $10^{14}$ per square centimeter per second ${ }^{2 n}$. Thus, instead of a timescale of 10 years for sputtering, we might expect a degradation timescale of a few days for atomic oxygen. Indeed, in LEO, only a few weeks are required to destroy a 1 mil layer of a highly reactive material like Kapton ${ }^{13}$. However, not all materials are reactive in atomic oxygen. For missions with LEO orbit times of more than a few days, surface materials that are non-reactive must be used.

Eck and his associates at CWRU ${ }^{28}$, as well as the author ${ }^{\infty}$, Banks $^{30}$, and others have measured AO reaction rates and kinetics for a variety of spacecraft materials. Atomic oxygen durability of materials and coatings continues to be investigated in ground tests and the recent EOIM-3 Shuttle flight experiment. EOIM-3 evaluated a host of spacecraft surface materials for atomic oxygen durability in LEO in midyear, 1992. A list of materials flown as part of the SP-100 program alone is given in Table I. EOIM-3 samples are now undergoing mass loss and surface property tests to determine their reactivity in LEO conditions.

\section{$\underline{X}$. Lunar and Planetary Interactions}

Finally, evaluation of the interactions of spacecraft and their power systems with lunar and planetary environments has started. A Workshop on Chemical and Electrical Interactions on Mars was held at NASA LeRC on November 19 and 20, 1991. Many of the primary interactions were identified. In Low Mars Orbit, many of the concerns now being addressed for LEO will be important, including AO degradation and arcing to the plasma. The present LEO environmental interactions investigations will be very relevant to these issues. On the surfaces of the moon and Mars, new issues arise, such as Paschen breakdown in low pressure neutral environments, chemical and electrical interactions with dust, etc. A workshop report has been published, giving a first evaluation of important interactions ${ }^{14}$.

\section{Summary and Recommendations}

Wherever there are discontinuous structure potentials of more than $100 \mathrm{~V}$ on spacecraft, they must be connected with an insulating material of sufficient thickness to stand off the potential difference.

Particular attention must be paid to geometries and materials where there are exposed conductor-insulator junctions in LEO. In particular, conductors known to have a plasma arcing threshold higher than the conductor potential relative to the surrounding plasma must be used. Plasma testing to identify such materials is suggested.

There is some evidence that arc rates are higher for materials that adsorb water and/or other volatile materials. Such materials should be avoided near conductor-insulator junctions, such as the SP100 UIM joint or solar cell edges on solar powered spacecraft. Geometry can influence plasma arcing as well, and geometries that help prevent ions from entering the conductor-insulator region are preferred.

For LEO missions, spacecraft surfaces should avoid exposed conductors, or at least exposed conductor-insulator junctions, to avoid arcing. For GEO missions, fully conductive surfaces should be used everywhere to avoid differential charging.

For LEO missions, it is recommended that sulfuric acid anodization be used as an insulating surface in regions where thermal control is not an overriding concern, and that $A O$ protected aluminized kapton blankets (kapton surface on the outside) of $1000 \mathrm{~V}$ dielectric strength be used in all other outside surfaces of a high voltage spacecraft. 
Whenever long life is required in a high plasma density environment such as low planetary orbits, surfaces should be insulated to prevent sputtering of high potential conductors in the plasma.

Where micrometeoroids, debris, or manufacturing defects produce small pinholes in insulators, the sputtering rate will be greatly enhanced by ion focusing onto the pinhole. Underlying conductors must be of sufficient thickness to withstand puncture at accelerated sputtering rates. Materials of low sputtering rate also may be used, if they satisfy thermal, atomic oxygen, and other requirements. Optical, thermal, or other specialized coatings in the line of sight to the sputtering pinhole will be coated with the sputtered material, over a long period of time. It is desirable to place such surfaces so they have no direct line of sight to surfaces that might undergo sputtering.

For missions with LEO orbit times of more than a few days, surface materials that are nonreactive with high energy atomic oxygen must be used. Designs must also take account of debris impacts and radiation damage.

In Low Mars Orbit, many of the concerns now being addressed for LEO will be important, including $A O$ degradation and arcing to the plasma. The present LEO environmental interactions investigations will be very relevant to these issues. On the surface of Mars, new issues arise, such as Paschen breakdown in low pressure neutral environments, chemical and electrical interactions with dust, etc. Similar issues may also arise on the Moon. For such missions, new environmental interactions concerns must be addressed as they are studied further.

\section{References}

${ }^{1}$ Vampola, A.L. (1980), "Radiation Effects on Space Systems and Their Modeling", in Space Systems and Their Interactions with Earth's Space Environment, Progress in Astronautics and Aeronautics, Vol. 71, New York: AIAA, H.B. Garrett and C.P. Pike, eds., p. 339.

${ }^{2}$ Frezet, M., Daly, E.J., Granger, J.P., and Hamelin,
J. (1989), "Assessment of Electrostatic Charging of Satellites in the Geostationary Environment", ESA Journal, Vol. 13, p. 91.

${ }^{3}$ Winningham, D. (1992), SouthWest Research Institute, Private Communication.

${ }^{4}$ Moorehead, R. (1992), NASA SSF Program Office, Private Communication.

${ }^{5}$ Herr, J.L. (1991), "A Charging Study of ACTS Using NASCAP", NASA CR-187088.

'Ferguson, D.C. (1986), The Voltage Threshold for Arcing for Solar Cells in LEO - Flight and Ground Test Results", NASA TM-87259.

'Purvis, C.K, Garrett, H.B., Whittlesey, A., and Stevens, N.J. (1984), "Design Guidelines for Assessing and Controlling Spacecraft Charging Effects", NASA TP-2361.

"Ferguson, D.C. (1989), "Solar Array Arcing in Plasmas",in the Proceedings of the Third Annual Workshop on Space Operations, Automation and Robotics (SOAR '89) S. Griffin, ed., NASA CP3059, p. 509.

'Jongeward, G.A., Katz, I., Kuharski, R.A., and Lilley, J.R., Jr. (1990), "Analysis of SP-100 Environment Interactions II", S-Cubed Div. of Maxwell Labs., Contract Report SSS-DTR-90-11284, W/O 11259, under NASA LeRC Contract NAS3-23881.

${ }^{10}$ Ferguson, D.C., Snyder, D.B., and Carruth, R. (1990), "Findings of the Joint Workshop on Evaluation of Impacts of Space Station Freedom Ground Configurations", NASA TM-103717.

${ }^{11}$ Snyder, D.B. (1991), NASA LeRC, Private Communication.

${ }^{12}$ Stevens, N.J. (1978), "Interactions Between Spacecraft and the Charged Particle Environment", in Spacecraft Charging Technology 1978, NASA CP2071, pp. 268-294.

${ }^{13}$ Ferguson, D.C. (1990), "Atomic Oxygen Effects on Refractory Materials", in Materials Degradation in Low Earth Orbit (LEO), Warrendale, Pa: TMS, V. Srinivasan and B.A. Banks, eds., p. 100. 
14Kolecki, J.C., and Hillard, G.B. (1992), Proceedings of the Electrical and Chemical Interactions at Mars Workshop, Nov. 19-20, 1991, NASA CP-10093.

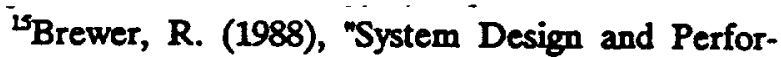
mance Characteristics", in SP-100 Reference Flight System System Desien Review, Volume 1. Systems, 17-19 May 1988, General Electric, p. 1.8-31.

1G Upschulte, B.L., Weyl, G.M, Marinelli, WJ., Aifer, E. Hastings, D., and Snyder, D. (1991), "Significant Reduction in Arc Frequency of Negatively Biased Solar Cells: Observations, Diagnostics, and Mitigation Technique(s), in the Proceedings of the Space Photovoltaic Research and Technology Conference 1991 (SPRAT 91). NASA CP-3121, p. 32-1.

${ }^{17}$ Katz, I. (1989), U.S. Patent \# 4835341 issued to Maxwell Laboratories.

${ }^{18}$ Grier, N.T. and Domitz, S. (1991), in the Proceedings of the Fifth Annual Workshop on Space Operations, Applications and Research (SOAR 91) K Krishen, ed., NASA CP-3127, p. 703.

${ }^{19}$ Kessler, D.J. (1988), "Orbital Debris Environment and Data Requirements", in NASA/SDIO Space Environmental Effects on Materials Workshop, NASA CP-3035, pp. 281-300.

${ }^{20}$ Vampola, A.L. (1988), The Space Particle Environment", in NASA/SDIO Space Environmental Effects on Materials Workshop, NASA CP-3035, pp. 367-382.

${ }^{2}$ Slemp, W.S. (1988), "Ultraviolet Radiation Effects", in NASA/SDIO Space Environmental Effects on Materials Workshop NASA CP-3035, pp. 425446.

${ }^{2}$ Eck, T.G., Chen, L.-Y., and Hoffman, R.W. (1991), "Sputtering of Ions from Cu and Al by Low Energy Oxygen Ion Bombardment", in the Proceedings of the Fifth Annual Workshop on Space Operations. Applications and Research (SOAR '91) K. Krishen, ed., NASA CP-3127, p. 716.
${ }^{23}$ Eck, T.G. (1993), to be published.

${ }^{21}$ Herr, J.L. and Snyder, D.B. (1993), "Plasma Sheath Effects on Ion Collection by a Pinhole", presented at the 31st AIAA Aerospace Sciences Mtg., Reno, NV, Jan. 11-14, 1993.

${ }^{25}$ Snyder, D.B. and Herr, J.L. (1991), in the Proceedings of the Fifth Annual Workshop on Space Operations. Applications and Research (SOAR 91). K Krishen, ed., NASA CP-3127, p. 694.

${ }^{26}$ Grier, N.T. and Domitz, S. (1989), Measured Current Collection for High Temperature and High Voltage Power Cables Used in Earth Orbit", presented at the Aerospace Sciences Meeting of the AIAA, Reno, Nevada, January.

${ }^{2}$ Tennyson, R.C., and Morison, W.D. (1990), in Materials Deoradation in Low Earth Orbit (LEO), Warrendale, Pa: TMS, V. Srinivasan and B.A. Banks, eds., p. 59.

${ }^{20}$ Horton, C.C., Eck, T.G., and Hoffman, R.W. (1989), "Pulsed Ion Beam Investigation of the Kinetics of Surface Reactions", J. Vac. Sci. Technol. A. 7, pp. 2143-2146.

${ }^{20}$ Ferguson, D.C. (1984), "The Energy Dependence and Surface Morphology of Kapton Degradation Under Atomic Oxygen Bombardment", in Proceedings of the 13th Space Simulation Conference, NASA CP-2340, p. 205.

${ }^{30}$ Banks, B.A., Auer, B.M., and DiFilippo, F. (1990), "Atomic Oxygen Undercutting of Defects on SiO2 Protected Polyimide Solar Array Blankets", in Materials Degradation in Low Earth Orbit (LEO), Warrendale, Pa: TMS, V. Srinivasan and B.A. Banks, eds., p. 15. 
TABLE I

SP-100 SAMPLES ON EOIM-3 SHUTTLE FLIGHT EXPERIMENT

\author{
Niobium-1 Zirconium \\ Tungsten-Niobium Composite \\ PWC-11 \\ Rokide C \\ Europium Oxide \\ Indium Tin Oxide \\ Molybdenum \\ 304 Stainless Steel \\ Udimet 720 \\ Polycarbonate \\ FEP on Kapton \\ Space Suit Outer Fabric \\ FEP \\ Kevlar
}

\author{
Inconel 718 \\ Molybdenum-13 Rhenium \\ GE Black \\ BN Point Type A \\ Brass \\ Tungsten \\ 6061-T6 Aluminum \\ Diamond-like Film \\ Poly Mesh \\ Viton Fabric \\ Kapton \\ Space Suit Fabric Upside Down \\ Titanium \\ Nomex
}

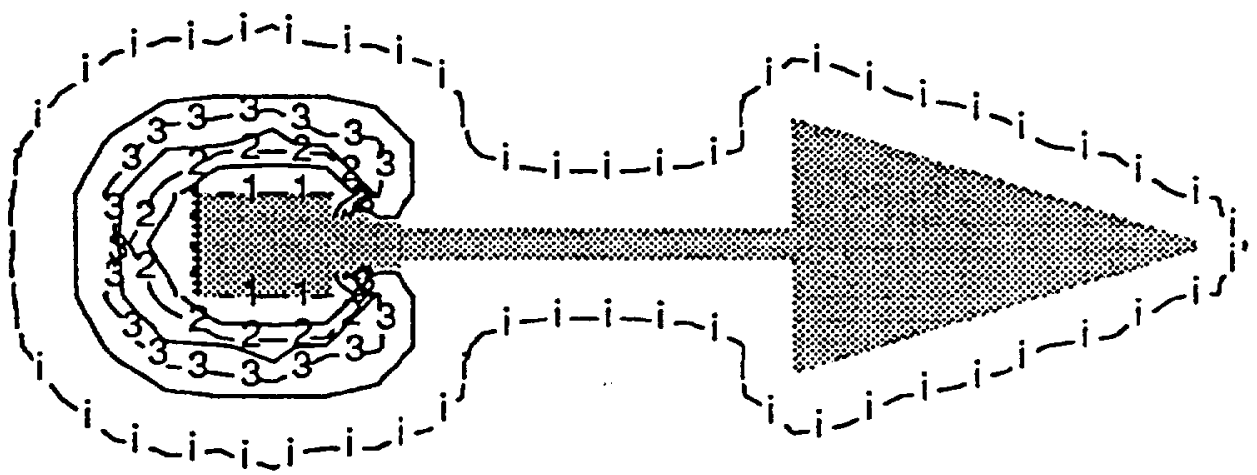

Figure 1. Equipotential contours around SP-100 payload (to left) and power system (right) computed with NASCAP/LEO. 


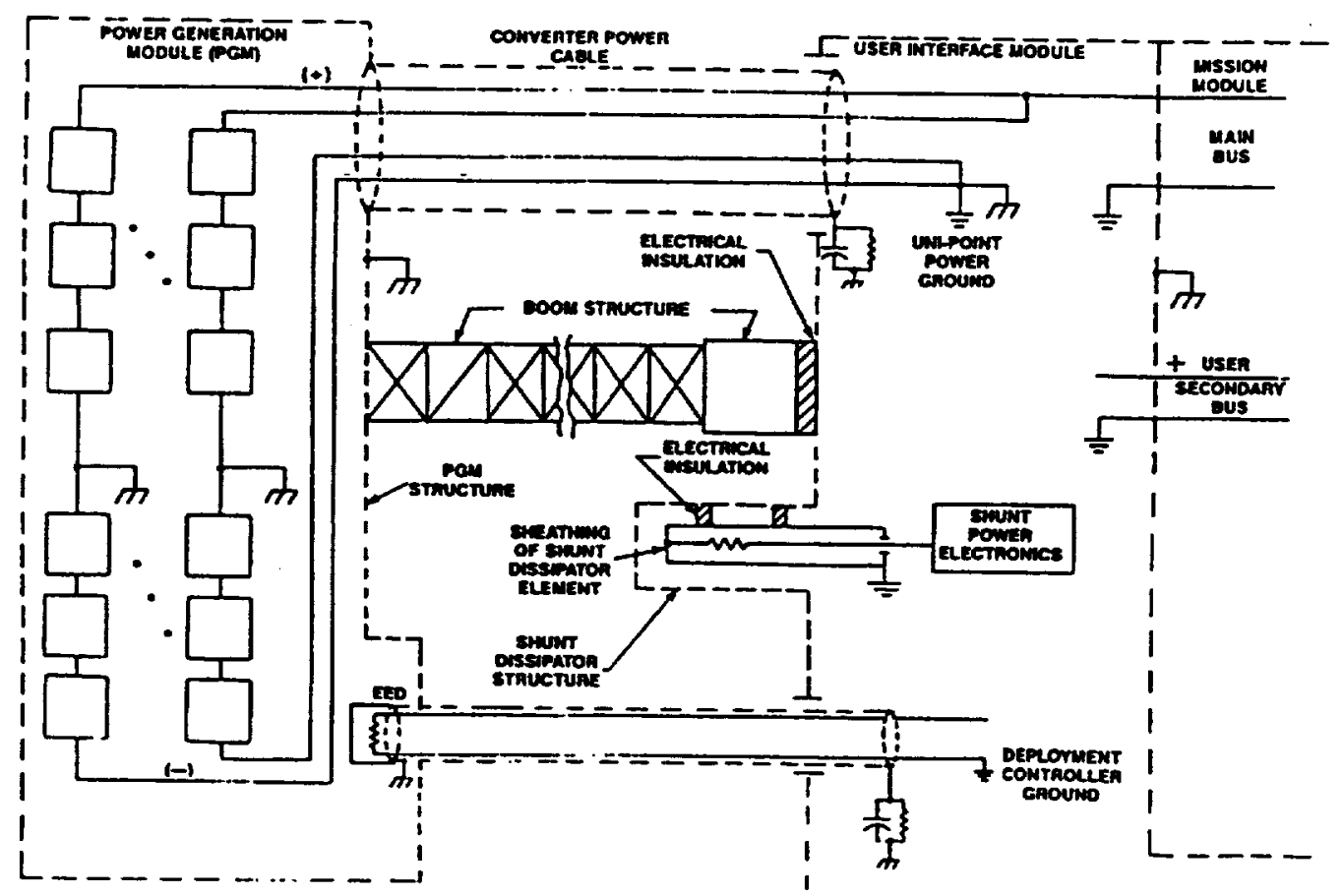

Figure 2. The grounding scheme utilized by SP-100, with the payload at right, and the power system at left. Notice the difference in grounding of the payload and power modules.

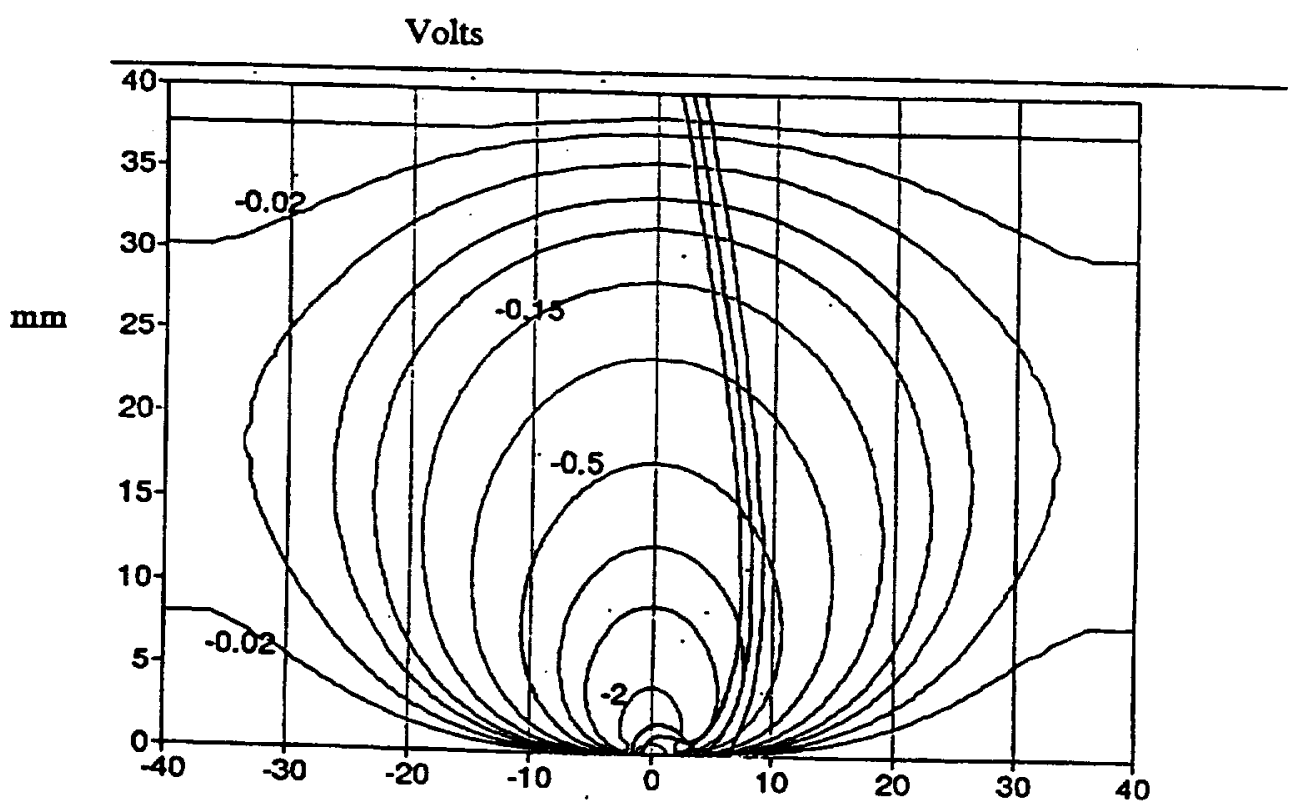

Figure 3. Equipotential contours and ion tracks above a $1 \mathrm{~mm}$ pinhole in a perfect insulator, underlying conductor biased to $-140 \mathrm{~V}$. 


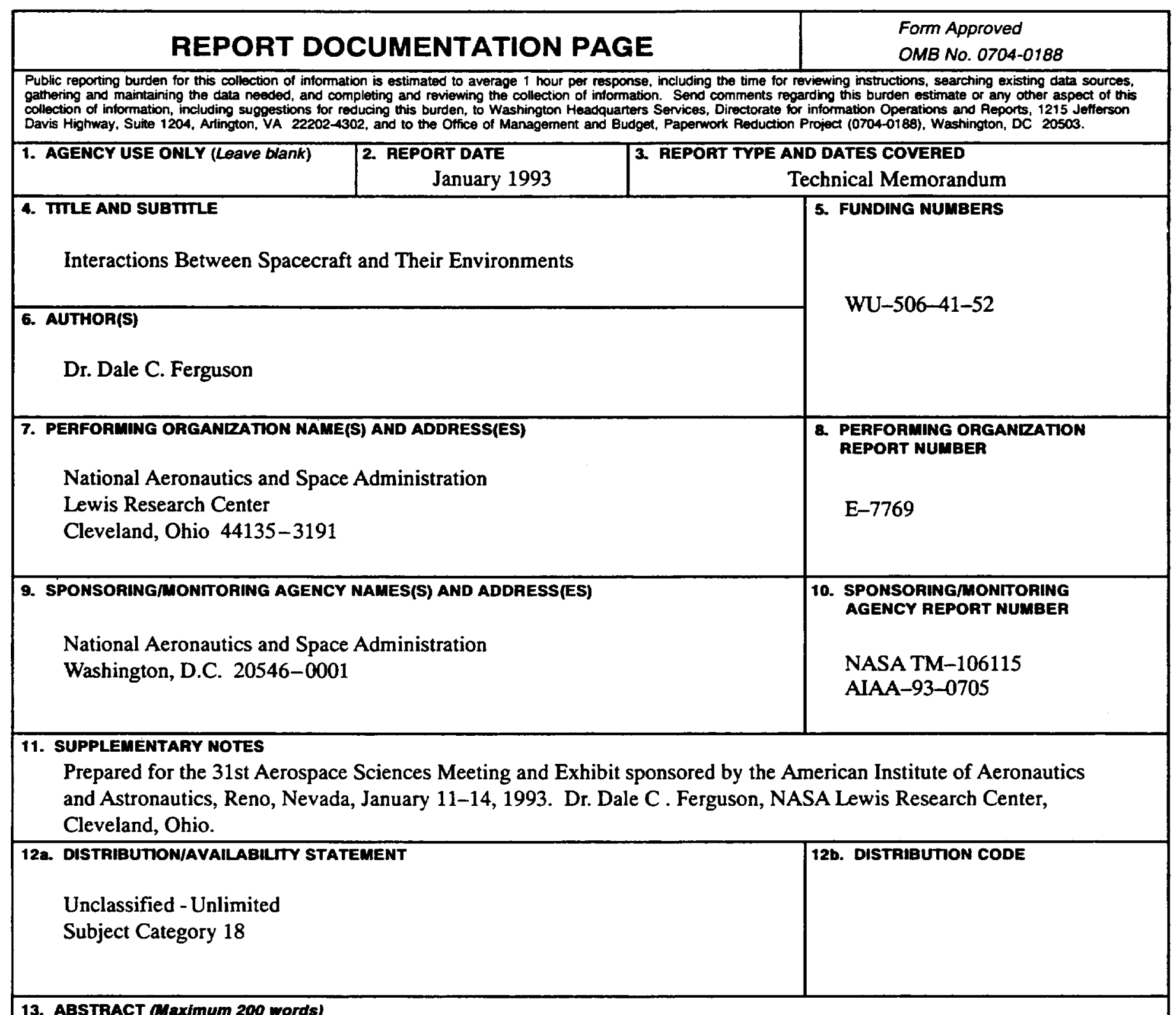

13. ABSTRACT (Maximum 200 words)

Spacecraft inevitably interact with their environments. Besides the interactions one immediately thinks of in space (zero-g, solar heating, atmospheric drag, expansion into vacuum conditions, etc.) other interactions are also important. Those of interest to spacecraft designers so far may be grouped under several headings; plasma interactions and spacecraft charging, impact of debris and micrometeoroids, chemical reactions with neutral species, radiation degradation, etc. Researchers have made great progress in defining and evaluating the interactions of spacecraft with their expected ambient environments near Earth and in interplanetary space. Here we discuss some of these interactions with an eye toward expanding our knowledge into new environments, such as may be found at the moon and Mars, that will interact in new and different ways with exploring spacecraft and spacefarers.

\begin{tabular}{|c|c|c|c|}
\hline \multirow{2}{*}{\multicolumn{3}{|c|}{ Spacecraft; Environments; Interactions }} & $\begin{array}{c}\text { 15. NUMBER OF PAGES } \\
12\end{array}$ \\
\hline & & & $\begin{array}{r}\text { 16. PRICE CODE } \\
\text { A02 }\end{array}$ \\
\hline $\begin{array}{l}\text { 17. SECURITY CLASSIFICATION } \\
\text { OF REPORT } \\
\text { Unclassified }\end{array}$ & $\begin{array}{l}\text { 18. SECURITY CLASSIFICATION } \\
\text { OF THIS PAGE } \\
\text { Unclassified }\end{array}$ & $\begin{array}{l}\text { 19. SECURTYY CLASSIFICATION } \\
\text { OF ABSTRACT } \\
\text { Unclassified }\end{array}$ & 20. LUMITATION OF ABSTRACT \\
\hline NSN 7540-01-280-5500 & & & $\begin{array}{l}\text { andard Form } 298 \text { (Rev. 2-89) } \\
\text { escribed by ANSI Std. Z39-18 } \\
8-102\end{array}$ \\
\hline
\end{tabular}




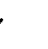

